\title{
Machine Parameters Monitoring For Automatic Plant Irrigation System Using GSM
}

\author{
Ms.Mohite Sayali U. ${ }^{1}$, Ms.Kumthekar Aarti. ${ }^{2}$ \\ BE. Student E\&TC, Dr. Daulatrao Aher College of Engg. Karad, India ${ }^{1}$ \\ Assistant professor, E\&TC Department, Dr. Daulatrao Aher College of Engg. Karad, India ${ }^{2}$
}

\begin{abstract}
Now a days farmers are struggling hard in the agricultural fields round the clock. They do their field work in the morning session and irrigate their land during night time with intermittent intervals. Farmers working in the farm lands are dependent on the rains and boar wells for the irrigation of land. Even if farm land has water pump, manual intervention by farmers is required to turn ON/OFF pump whenever needed. The task of irrigating fields is becoming quite difficult for the farmers due to lack of regularity in their work and negligence on their part because sometimes they switch on the motor and then forget to switch of which may lead to wastage of water. The proposed system, tried to minimize manual intervention by the farmer and helps the farmer to ON/OFF the motor and provide update status of the operation carried out in the agricultural fields via SMS with the help of GSM modem and monitor Temperature, Voltage, Current and Dry run of the motor because farmers are not familiar with the technical problems occurred in the motor.
\end{abstract}

Keywords: Irrigation, Motor (Water pump), Sensors, Arduino Atmega328, GSM.

\section{I.INTRODUCTION}

Agricultural field is playing a vital role in Indian economy. Where irrigation mechanism is important. In India most of the irrigation are operated manually. In recent times, the farmers have been using irrigation technique through the manual control in which the farmers irrigate the land at regular intervals by turning the water pump ON/OFF when required but sometimes task of irrigating fields is becoming difficult due to irregularity in their work. The farmers does not get current situations of the agricultural land when they are not physically present in the land.[1] In this paper GSM(Global System for Mobile Communication) is used to ON/OFF the motor without physical presence of farmer in the field and inform the user about the exact field condition then the information is passed to user in the form of SMS. The proposed system monitors the Temperature, Voltage, Current and Dry run of the motor (water pump). In this controller act as a core device for functioning of the automated process

after it has been initiated by the GSM. The microcontroller continuously receives the data from sensors and after this the data is displayed on LCD. By giving miscall, water pump gets started, only when there is sufficient water level in the tank. For sensing the moisture level in soil we are using moisture sensor. When there is moisture in soil then the output of moisture sensor is low, otherwise its output is high. Once the motor is started, a constant monitoring on soil moisture and water level is done and once this moisture is reached to sufficient level, the motor is automatically turned off and for this the message is send to subscriber that the is turned off. Motor used for irrigation purpose need to be monitor for effective irrigation and protect it from damage. An easy way to comply with the conference paper formatting requirements is to usethis document as a template and simply type your text into it.

\section{II.LITERATURE SURVEY}

1. In the existing system farmers have to travels to fields often at odd hours just to switch ON/OFF the motor. Sometimes they forget to switch off and water pumps are left running for longer time. This lead to wastage of both electricity and water. Laxmi Shabadi proposed wireless connection between server and nodes. Irrigation will takes place only when there will be intense requirement of water.[1]

2. In Purnima, S.R.N Reddy, "Design of remote monitoring and controlling system with automatic plant irrigation system using GSM-Bluetooth", proposed system is artificially supplied water to land where crops are cultivated. Traditionally boar wells, canal water \& rainfall were a major source of water supply for irrigation. This method has led to several drawbacks like under irrigation, occur irrigation which in turn causes leaching and loss of nutrient content of soil.[2]

3. In past few years controlling and monitoring the machines remotely has seen an interesting field of study among researchers. This paper mainly focuses on reviews in the field of remote monitoring and control, the technology used and their potential advantages. The paper proposes an innovative GSM/Bluetooth based remote controlled embedded system for irrigation.[3] 
4. As per Shweta hugar, Basavaraj Amarapur to protect an level to turn on the motor. When there is moisture in soil Induction motor easily, a microcontroller based fault then output of moisture sensor is low, water pump is in off detection and protection of Induction motor proposed. condition and there is sufficient level of water to turn on This system tends to develop for protection of three phase motor. If any fault is detect then it will send SMS to user induction motor from over voltage and under voltage, over for correct the fault.

current, over speed, temperature, Line frequency and phase failure with their sensing circuits [4].

B. Hardware Details:

5. This automated irrigation system based on the wireless sensor network with GSM-BLUETOOTH for control of irrigation and real time monitoring of agriculture. For real time monitoring of crops sensors are installed and these are controlled via SMS by using GSM module. This SMS shared via GSM/BLUETOOTH and interfaced with the main microcontroller. The microcontroller controls desired operation at the farm. This system also informs about temperature, soil moisture to the farmers by SMS via GSM BLUETOOTH module. According to this information action are taken by the farmer.[5]

\section{III.PROPOSED METHODOLOGY}

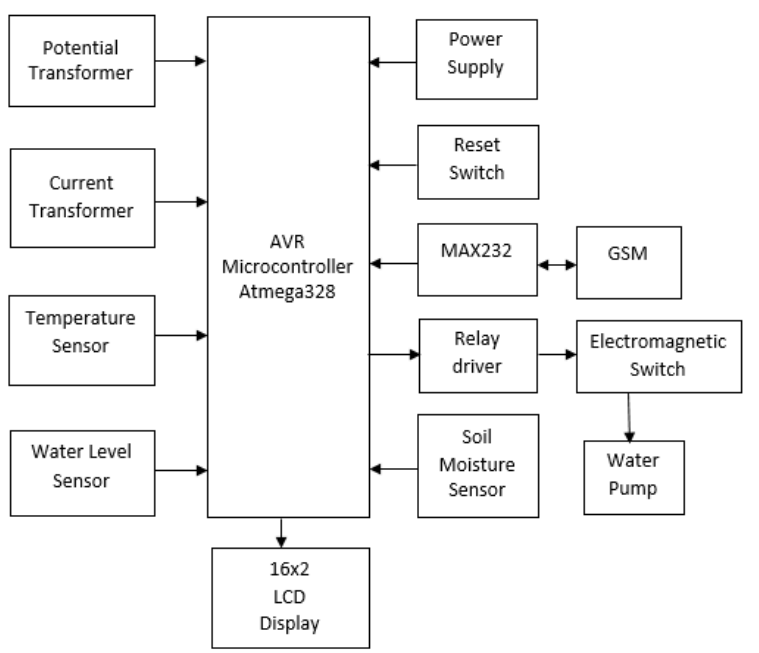

Fig.1 Block Diagram of Proposed System.

A.Working Principle:

The proposed system monitors parameters like Temperature, Voltage, Current and Dry run of the motor via LCD display. In this system Potential transformer is used to sense voltage variation whereas current transformer is used to detect the over load/over current of the motor. LM35 is a temperature sensor is usually analog sensor which is used to sense temperature and convert it to voltage level. It is helps to detect over heat protection. Water level sensor protects motor from Dry run condition and it avoid no load. This all data is converted to proper value by using signal condition and it given to AVR Microcontroller for further process. AVR microcontroller is used as main decision device. It sense data from various sensors and take decision. Max232 level converter are used for serial communication in between AVR microcontroller and GSM module. If GSM module detect SMS from user then it first check soil moisture and water

(a).Atmega328:

The Atmega328 is a low-power CMOS 8-bit microcontroller based on the AVR RISC architecture. By executing powerful instructions in a single clock cycle, the ATmega 8 achieves throughputs approaching 1 MIPS per $\mathrm{MHz}$, allowing the system designer to optimize power consumption versus processing speed.

Features

•High-performance, $\quad$ Low-power $\quad$ AVR ${ }^{\circledR} \quad$ 8-bit

Microcontroller

- $\mathrm{I} / \mathrm{O}$ and Packages

- 23 Programmable I/O Lines

- 28-lead PDIP, 32-lead TQFP,28-QFN/MLF and 32-pad QFN/MLF

-Operating Voltages

$-1.8-5.5 \mathrm{~V}$

-Speed Grades

-0 - $4 \mathrm{MHz} @ 1.8-5.5 \mathrm{~V}$

-0 - $10 \mathrm{MHz} @ 2.72-5.5 \mathrm{~V}$

-Power Consumption at $1 \mathrm{MHz}, 1.8 \mathrm{~V}, 25^{\circ} \mathrm{C}$

- Active Mode: $0.2 \mathrm{~mA}$

- Power-down Mode:0.1 $\mu \mathrm{A}$

- Power-save Mode: $0.75 \mu \mathrm{A}$

(b)GSM Module:

GSM is mobile communication modem. It stands for global system for mobile communication. GSM modem is a specialized type of wireless modem. A GSM modem accepts a SIM card and operates just like a mobile phone because it is wireless network. A GSM modem works as like of the dial-up modem.GSM module (SIM 900) is used to send \& receive the SMS from/to user. It is an AT command supportable serial communication module with 9600 default band rate. It works over the band of $900 \mathrm{MHz}$ to $1800 \mathrm{MHz}$.

\section{Feature:}

$\square$ High Quality Product (Not hobby grade)

Plug and Play Module

Tri-Band GSM/GPRS 900/ 1800/ $1900 \mathrm{MHz}$

$5 \mathrm{~V}$ TTL interface for direct connection with MCU/Arduino

Configurable baud rate

SMA connector with GSM Antenna.

Built in SIM Card holder.

Built in Network Status LED

Inbuilt Powerful TCP/IP protocol stack for internet data transfer over GPRS.

Standard $2.54 \mathrm{~mm}$ Connector Pitch 
International Advanced Research Journal in Science, Engineering and Technology

National Conference on Emerging trends in Electronics \& Telecommunication Engineering (NCETETE 2017) AGTI's Dr. Daulatrao Aher College Engineering, Vidyanagar Extension, Karad

Vol. 4, Special Issue 2, January 2017

Switch ON/OFF Pin at connector

Status LED Pin at connector

Hardware Flow controlling pins available at connector

\section{(c).MAX232}

The MAX 232 is an IC, first created in 1987 by Maxim Integrated Products, that converts single from anRS-232 serial port to signals suitable for use in TTL compatible digital logic circuits. The MAX232 is a dual driver/receiver and typically convert the RX,TX,CTS and RTS signals. It is helpful to understand what occurs to voltage levels.

When a MAX232 IC receives a TTL level to convert, it changes a TTL logic 0 to between +3 and $+15 \mathrm{v}$, and changes TTL logic 1 to between -3 to $-15 \mathrm{~V}$, and vice versa for converting from RS232 to TTL. This can be confusing when you realize that the RS232 data transmission voltages at a certain logic state are opposite from the RS232 control line voltages at the same logic state.

(d). LM35 Temperature Sensors:

The LM35 is an integrated circuit sensor that can be used to measure temperature with an electrical output proportional to the temperature (in degree Celsius).It is semi-conductor device whose resistance changes according to change in temperature in some cases the resistance or a thermistor at room temperature may decrease as much as 5 percent for each $1^{0} \mathrm{C}$ rise in temp. This high sensitivity to temp changes makes thermistor extremely well suited to precision temp measurement, Control and compensation. Thermistor are therefore widely used in such apparatus, Specially in the lower temp range of $-100{ }^{0} \mathrm{C}$ to $300{ }^{\circ} \mathrm{C}$.

(e).Water Level Sensor:

Basically it is a reed type of switch. Reed switch sensors contain hermetically sealed reed elements which can be open or closed in their normal state. When a magnetic field moves within proximity of the switch, magnetism is induced in the leads and forces the contacts state, as shown in figure. This switch gives $0 \mathrm{v}$ when in open condition and $24 \mathrm{v}$ when switch get closed

\section{(f).Soil moisture sensor:}

The soil moisture sensor is used to measure water content of soil. This makes it ideal for performing experiment in courses such as soil science,agricultural science, botany and biology. The soil moisture sensor uses capacitance to measure a dielectric permittivity of the surrounding medium. In soil dielectric permittivity is a function of the water.

(g).Power supply:

Power supply is the major concern for every electronic device .Since the controller and other devices used are low power devices there is a need to step down the voltage and as well as rectify the output to convert the output to a constant dc.Power supply unit is the basic requirement for electronic devices.

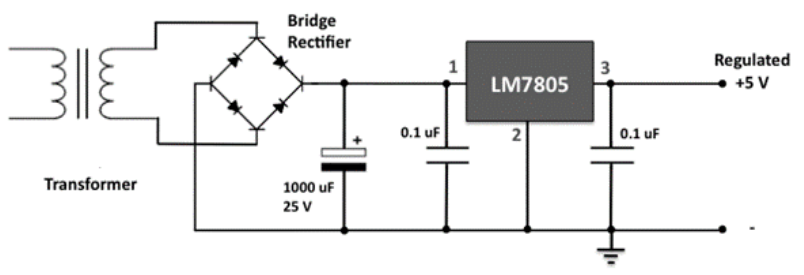

Fig. 2 Power Supply

The major blocks of power supply are given below

Transformer:

Transformer is a device used to increment or decrement the input voltage given as per the requirement. The transformers are classified into two types depending upon there functionality.

- Step up transformer

- Step down transformer

Here we use a step down transformer for stepping down the house hold ac power supply i.e. the 230-240V power supply .We use a 9-0-9V center tapped step down transformer.

Rectifier:

The output of the transformer is an ac and should be rectified to a constant dc for this it is necessary to feed the output of the transformer to a rectifier. The rectifier is employed to convert the alternating ac to a constant dc. There are many rectifiers available in the market some of them are:-

- Half wave rectifier

- Full wave rectifier

- Bridge rectifier

The rectification is done by using one or more diodes connected in series or parallel. If only one diode is used then only first half cycle is rectified and it is termed as half wave rectification and the rectifier used is termed as half wave rectifier. If two diodes are employed in parallel then both positive and negative half cycles are rectified and this is full wave rectification and the rectifier is termed as Full wave rectifier. If the diodes are arranged in the form of bridge then it is termed as Bridge rectifier, it acts as a full wave rectifier. These rectifiers are available in the market in the form of integrated chips (I.Cs).

Filter:

Capacitor filter is used to remove ac ripple content is the rectified output in order to smoothen output waveform.

7805 voltage regulator:

The voltage regulator is used for the voltage regulation purpose. We use IC 7805 voltage regulator. The IC number has a specific significance. The number 78 
represents the series while 05 represent the output voltage generated by the IC

C. Software details:

(a).Keil Compiler:

The keil c compiler for the microcontroller is most popular compiler in the world. It provides more features than another $8051 \mathrm{C}$ compiler available today. The C51 compiler allows you to write 8051 microcontroller applications in $\mathrm{C}$ that, once compiled, have the efficiency and speed of assembly language.

(b).Proteus 8 Professional:

Proteus is software for microprocessor simulation, schematic capture, and printed circuit board (PCB) design. It is developed by Lab center Electronics.Proteus 8 is best simulation software for various designs with microcontroller. It is mainly popular because of availability of almost all microcontrollers in it. So, it is a handy tool to test programs and embedded designs for electronics hobbyist. You can simulate your programming of microcontroller in Proteus 8 simulation software. continuously monitoring the status of motor parameters and the soil moisture, we can control the flow of water and thereby reduce the wastage. These all system are then controlled by sending SMS from our mobile.

\section{REFERENCES}

[1] Laxmi shabadi, Nandini patil, Nikita.M, Shruti.J, Smitha.P and Swati.C, "Irrigation control system using android and GSM for efficient use of water and power. International journal of advanced research in computer science and software engineering, volume 4, Issue 7, July 2014

[2] Purnima, S.R.N Reddy, "Design of Remote Monitoring \& control system with automatic irrigation system using GSM. Bluetooth, on IJCA, 2012

[3] Indu Gautma, S.R.N. Reddy, "Innovative GSM Bluetooth Based Remote Controlled Embedded System for Irrigation", on IJCA, June 2012

[4] Chaitanya N.Jibhakateand Mrs.Vijaya Huchche "Speed Control of Single Phase Induction Motor Using Micro-Controller" IJERA pp.29-32 April2014

[5] Rashid Hussain, JL Sahgal, Anshulgangwar, Md.Riyaj, "Control of Irrigation Automatically By Using Wireless Sensor Network",International Journal of Soft Computing and Engineering (IJSCE), ISSN: 2231-2307, Volume-3, Issue-1, March 2013.

\section{IV.FLOW CHART:}

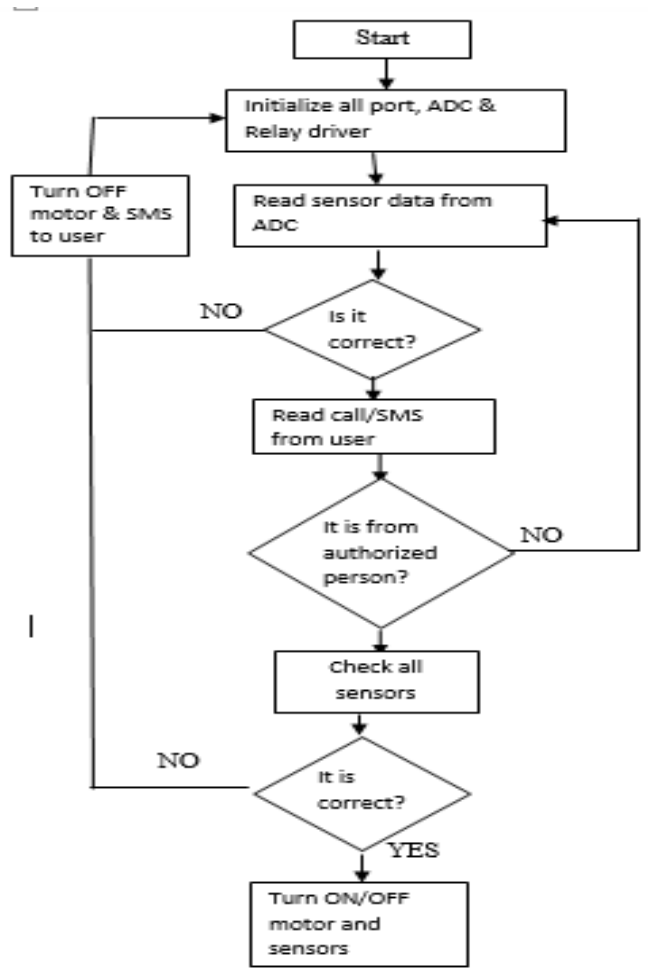

V. CONCLUSION

Irrigation has been the backbone of human civilization since man has started agriculture. As the generation involved, man developed many methods of irrigation to supply water to the land. Present work is attempt to save the natural resources available for human kind. By 